\title{
Ear decomposition and balanced neighborly simplicial manifolds
}

\author{
Hailun Zheng \\ Department of Mathematics \\ University of Michigan \\ Ann Arbor, MI, 48109, USA \\ hailunz@umich.edu
}

Submitted: Nov 26, 2018; Accepted: Dec 22, 2019; Published: Jan 10, 2020

(C) The author. Released under the CC BY-ND license (International 4.0).

\begin{abstract}
We find the first non-octahedral balanced 2-neighborly 3-sphere and the balanced 2-neighborly triangulation of the lens space $L(3,1)$. Each construction has 16 vertices. We show that there exists a balanced 3-neighborly non-spherical 5manifold with 18 vertices. We also show that the rank-selected subcomplexes of a balanced simplicial sphere do not necessarily have an ear decomposition.
\end{abstract}

Mathematics Subject Classifications: 05E45

\section{Introduction}

A simplicial complex is called $k$-neighborly if every subset of vertices of size at most $k$ is the set of vertices of one of its faces. Neighborly complexes, especially neighborly polytopes and spheres, are interesting objects to study. In the seminal work of McMullen [12] and Stanley [19], it was shown that in the class of polytopes and simplicial spheres of a fixed dimension and with a fixed number of vertices, the cyclic polytope simultaneously maximizes all the face numbers. The $d$-dimensional cyclic polytope is $\left\lfloor\frac{d}{2}\right\rfloor$-neighborly. Since then, many other classes of neighborly polytopes have been discovered. We refer to [4], [16] and [18] for examples and constructions of neighborly polytopes. Meanwhile, the notion of neighborliness was extended to other classes of objects: for instance, neighborly cubical polytopes were defined and studied in [8], [9], and [17], and neighborly centrally symmetric polytopes and spheres were studied in [1], [3], [7] and [14].

In this paper we discuss a similar notion for balanced simplicial complexes. Balanced complexes were defined by Stanley in [20], where they were called completely balanced. A $(d-1)$-dimensional simplicial complex is called balanced if its graph is $d$-colorable. For instance, the barycentric subdivision of regular CW complexes and order complexes are 
balanced. We say that a balanced simplicial complex is balanced $k$-neighborly if every set of $k$ or fewer vertices with distinct colors forms a face. For example, if $\Delta_{1}$ and $\Delta_{2}$ are balanced $k$-neighborly spheres, then the join $\Delta=\Delta_{1} * \Delta_{2}$ is also a balanced neighborly $k$-sphere, and we call $\Delta$ join-decomposable. However, apart from the cross-polytopes, it is not known whether other join-indecomposable balanced $k$-neighborly polytopes or spheres exist. To the best of our knowledge, no examples of such objects appear in the current literature, even for $k=2$. As for balanced 2-neighborly manifolds, one such construction that triangulates the sphere bundle is given in [11]; it is also a minimal balanced triangulation of the underlying topological space.

This more or less explains why so far there is even no plausible sharp upper bound conjecture for balanced spheres or manifolds. The goal of this paper is to partially remedy this situation by searching for balanced neighborly spheres and manifolds of lower dimensions. It turns out that even in the lower dimensional cases balanced neighborly spheres or manifolds with a given number of vertices do not always exist.

- The octahedral 3-sphere is the only balanced 2-neighborly 3 -sphere with less than 16 vertices.

- There is a unique balanced 2-neighborly 4-sphere with 15 vertices, known as ${ }^{4} 15_{2}^{5}$ in [10].

- There exists a balanced 3-neighborly non-spherical 5-manifold with 18 vertices.

- There are two constructions of balanced 2-neighborly 3-manifolds with 16 vertices; one triangulates the sphere, and the other triangulates the lens space $L(3,1)$.

In a different direction, it is also interesting to ask whether every rank-selected subcomplex of a balanced simplicial polytope or sphere has a convex ear decomposition. This statement, if true, would imply that rank-selected subcomplexes of balanced simplicial polytopes possess certain weak Lefschetz properties, see Theorem 3.9 in [22]. As a consequence, it would also provide an alternative proof of the balanced Generalized Lower Bound Theorem, see Theorem 3.3 and Remark 3.4 in [13]. We present an example giving a negative answer to this question for 3-dimensional spheres.

The structure of this manuscript is as follows. In Section 2, after reviewing basic definitions, we establish basic properties of balanced neighborly spheres; in particular, we prove that for some values of $f_{0}$, such spheres cannot exist. In Section 3 we discuss how to find balanced $k$-neighborly $(2 k-1)$-manifolds from less neighborly balanced $(2 k-2)$ spheres, for $k=2,3$. In Section 4, we construct a balanced 2-neighborly 3-sphere with 16 vertices. In Section 5, we present the balanced 2-neighborly triangulation of $L(3,1)$ with 16 vertices. In Section 6 we provide a way to construct balanced spheres whose rank-selected subcomplex does not have an ear decomposition.

\section{Basic properties of balanced neighborly spheres}

A simplicial complex $\Delta$ with vertex set $V$ is a collection of subsets $\sigma \subseteq V$, called faces, that is closed under inclusion, and such that for every $v \in V,\{v\} \in \Delta$. For $\sigma \in \Delta$, let 
$\operatorname{dim} \sigma:=|\sigma|-1$ and define the dimension of $\Delta, \operatorname{dim} \Delta$, as the maximum dimension of the faces of $\Delta$. A facet is a maximal face under inclusion. We say that a simplicial complex $\Delta$ is pure if all of its facets have the same dimension.

If $\Delta$ is a simplicial complex and $\sigma$ is a face of $\Delta$, the star of $\sigma$ in $\Delta$ is $\operatorname{st}_{\Delta} \sigma:=\{\tau \in$ $\Delta: \sigma \cup \tau \in \Delta\}$. We also define the link of $\sigma$ in $\Delta$ as $\operatorname{lk}_{\Delta} \sigma:=\{\tau-\sigma \in \Delta: \sigma \subseteq \tau \in \Delta\}$, and the deletion of a subset of vertices $W$ from $\Delta$ as $\Delta \backslash W:=\{\sigma \in \Delta: \sigma \cap W=\varnothing\}$. If $\Delta_{1}$ and $\Delta_{2}$ are simplicial complexes on disjoint vertex sets, then the join of $\Delta_{1}$ and $\Delta_{2}$, denoted $\Delta_{1} * \Delta_{2}$, is the simplicial complex with vertex set $V\left(\Delta_{1}\right) \cup V\left(\Delta_{2}\right)$ whose faces are $\left\{\sigma_{1} \cup \sigma_{2}: \sigma_{1} \in \Delta_{1}, \sigma_{2} \in \Delta_{2}\right\}$.

If $\Delta$ is a pure $(d-1)$-dimensional complex such that every $(d-2)$-dimensional face of $\Delta$ is contained in at most two facets, then the boundary complex of $\Delta$ consists of all $(d-2)$-dimensional faces that are contained in exactly one facet, as well as their subsets. A simplicial complex $\Delta$ is a simplicial sphere (resp. simplicial ball) if the geometric realization of $\Delta$ is homeomorphic to a sphere (resp. ball). The boundary complex of a simplicial $d$-ball is a simplicial $(d-1)$-sphere. A simplicial sphere is called polytopal if it is the boundary complex of a convex polytope. For instance, the boundary complex of an octahedron is a polytopal sphere; we will refer to it as an octahedral sphere.

For a fixed field or group $\mathbf{k}$, we say that $\Delta$ is a $(d-1)$-dimensional $\mathbf{k}$-homology sphere if $\tilde{H}_{i}\left(\mathrm{lk}_{\Delta} \sigma ; \mathbf{k}\right) \cong \tilde{H}_{i}\left(\mathbb{S}^{d-1-|\sigma|} ; \mathbf{k}\right)$ for every face $\sigma \in \Delta$ (including the empty face) and $i \geqslant-1$. A homology $d$-ball (over $\mathbf{k}$ ) is a $d$-dimensional simplicial complex $\Delta$ such that (i) $\Delta$ has the same homology as the $d$-dimensional ball, (ii) for every face $F$, the link of $F$ has the same homology as the $(d-|F|)$-dimensional ball or sphere, and (iii) the boundary complex $\partial \Delta$ is a homology $(d-1)$-sphere. The classes of simplicial $(d-1)$-spheres and homology $(d-1)$-spheres coincide when $d \leqslant 3$. From now on all homology are computed with coefficients in $\mathbb{Z}$ and we will omit it from our notation.

Next we define a special structure that exists in some pure simplicial complexes.

Definition 1. An ear decomposition of a pure $(d-1)$-dimensional simplicial complex $\Delta$ is an ordered sequence $\Delta_{1}, \Delta_{2}, \cdots, \Delta_{m}$ of pure $(d-1)$-dimensional subcomplexes of $\Delta$ such that:

1. $\Delta_{1}$ is a simplicial $(d-1)$-sphere, and for each $j=2,3, \cdots, m, \Delta_{j}$ is a simplicial $(d-1)$-ball.

2. For $2 \leqslant j \leqslant m, \Delta_{j} \cap\left(\cup_{i=1}^{j-1} \Delta_{i}\right)=\partial \Delta_{j}$.

3. $\cup_{i=1}^{m} \Delta_{i}=\Delta$.

We call $\Delta_{1}$ the initial complex, and each $\Delta_{j}, j \geqslant 2$, an ear of this decompostion. Notice that this definition is more general than Chari's original definition of a convex ear decomposition, see [2, Section 3.2], where the $\Delta_{i}$ 's are required to be subcomplexes of the boundary complexes of polytopes. In particular, if a complex has no ear decomposition, then it has no convex ear decomposition. However, by the Steinitz theorem, all simplicial 2-spheres are polytopal, and hence also all simplicial 2-balls can be realized as subcomplexes of the boundary complexes of 3-dimensional polytopes. So for 2-dimensional 
simplicial complexes, the notion of an ear decomposition coincides with that of a convex ear decomposition.

A $(d-1)$-dimensional simplicial complex $\Delta$ is called balanced if the graph of $\Delta$ is $d$-colorable, or equivalently, there is a coloring map $\kappa: V \rightarrow[d]$ such that $\kappa(x) \neq \kappa(y)$ for any edge $\{x, y\} \in \Delta$. Here $[d]=\{1,2, \cdots, d\}$ is the set of colors. We denote by $V_{i}$ the set of vertices of color $i$. A balanced simplicial complex is called balanced $k$ neighborly if every set of $k$ or fewer vertices with distinct colors forms a face. We say $e$ is a missing colored edge if $e \notin \Delta$ and the vertices of $e$ have distinct colors. For $S \subseteq[d]$, the subcomplex $\Delta_{S}:=\{F \in \Delta: \kappa(F) \subseteq S\}$ is called the rank-selected subcomplex of $\Delta$. We also define the flag $f$-vector $\left(f_{S}(\Delta): S \subseteq[d]\right)$ and the flag h-vector $\left(h_{S}(\Delta): S \subseteq[d]\right)$ of $\Delta$, respectively, by letting $f_{S}(\Delta):=\#\{F \in \Delta: \kappa(F)=S\}$, where $f_{\varnothing}(\Delta)=1$, and $h_{S}(\Delta):=\sum_{T \subseteq S}(-1)^{\# S-\# T} f_{T}(\Delta)$. The usual $f$-numbers and $h$-numbers can be recovered from the relations $f_{i-1}(\Delta)=\sum_{\# S=i} f_{S}(\Delta)$ and $h_{i}(\Delta)=\sum_{\# S=i} h_{S}(\Delta)$.

In the remainder of this section, we establish some restrictions on the possible size of color sets of balanced neighborly spheres.

Lemma 2. Let $\Delta$ be a balanced $k$-neighborly homology $(2 k-1)$-sphere. Then $\Delta$ has the same number of vertices of each color. In particular, $f_{0}(\Delta)=2 k \ell$ for some $\ell \geqslant 2$.

Proof. Let $W \subseteq[2 k]$ be an arbitrary subset of the set of the colors with $|W|=k$. Since $\Delta$ is balanced $k$-neighborly, $\Delta_{W}$ is also balanced $k$-neighborly, and hence $\Delta_{W}$ is the join of $k$ color sets of colors in $W$, each considered as a 0 -dimensional complex. By the definition of the join and the flag $h$-numbers, we have $f_{U \cup\{i\}}(\Delta)=f_{U}(\Delta) f_{\{i\}}(\Delta)$ and hence $h_{U \cup\{i\}}(\Delta)=h_{U}(\Delta) h_{\{i\}}(\Delta)$ for all $i \in W, U \subset W$ and $i \notin U$. Therefore,

$$
\prod_{i \in W}\left(\left|V_{i}\right|-1\right)=\prod_{i \in W} h_{\{i\}}(\Delta)=h_{W}(\Delta) \stackrel{(*)}{=} h_{[2 k] \backslash W}(\Delta)=\prod_{i \in[2 k] \backslash W} h_{\{i\}}(\Delta)=\prod_{i \in[2 k] \backslash W}\left(\left|V_{i}\right|-1\right)
$$

where $(*)$ follows from the Dehn-Sommerville relations. Since $W$ is an arbitrary $k$-subset of $[2 k]$, it follows that each color set in $\Delta$ must have the same size.

Remark 3. Lemma 2 not only holds for homology $(2 k-1)$-spheres but also for orientable homology $(2 k-1)$-manifolds. Indeed by replacing the flag $h$-numbers with the flag $h^{\prime \prime}$ numbers (see [6] for definition), Theorem 4.1 in [6] gives $h_{W}^{\prime \prime}(\Delta)=h_{[2 k] \backslash W}^{\prime \prime}(\Delta)$, which further implies that $h_{W}(\Delta)=h_{[2 k] \backslash W}(\Delta)$ since both $W$ and $[2 k] \backslash W$ are of size $k$. The rest of the proof is the same.

Unfortunately, the above lemma is not sufficient to tell whether a balanced $k$-neighborly homology $(2 k-1)$-sphere or manifold with $2 k \ell$ vertices can exist for given $k, \ell \geqslant 2$.

\section{Balanced neighborly $(d-1)$-manifolds with $3 d$ vertices}

In this section, we consider balanced $\left\lfloor\frac{d}{2}\right\rfloor$-neighborly $(d-1)$-manifolds (for $d=3,4,5$ ) with each color set of size 3 . We begin with the following lemma. 

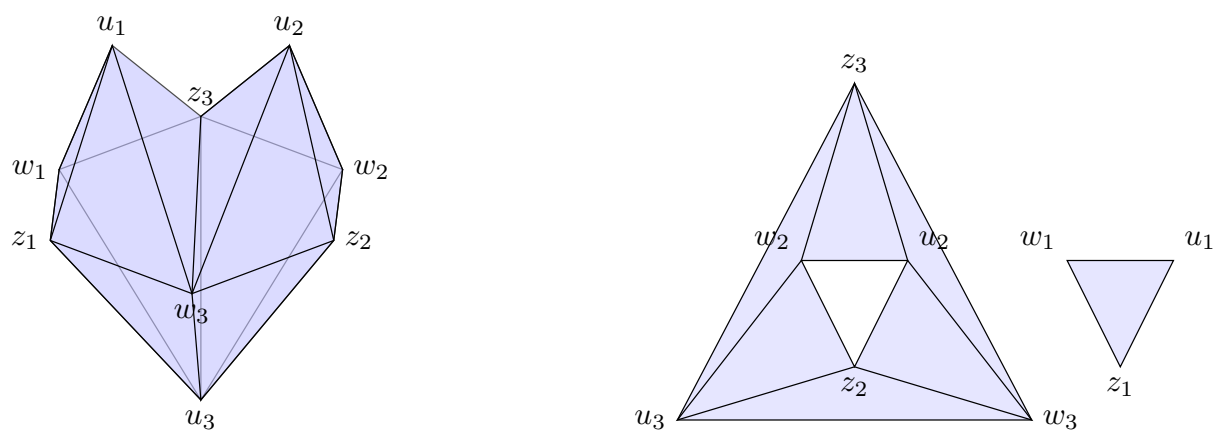

Figure 1: Left: triangulation of the vertex link $\mathrm{lk}_{\Delta} v_{i}$ for $v_{i} \in V_{4}$, where $\left\{u_{1}, u_{2}, u_{3}\right\}$, $\left\{w_{1}, w_{2}, w_{3}\right\}$ and $\left\{z_{1}, z_{2}, z_{3}\right\}$ are the three other color sets. Right: the complex $\Sigma$.

Lemma 4. Let $d \geqslant 4$. If $\Delta$ is a balanced homology $(d-1)$-sphere and $V_{d}=\left\{v_{1}, v_{2}, v_{3}\right\}$ is the set of vertices of color $d$, then $\mathrm{lk}_{\Delta} v_{i} \cap \mathrm{lk}_{\Delta} v_{j}$ is a homology $(d-2)$-ball for any $1 \leqslant i<j \leqslant 3$, and $\cap_{k=1}^{3} \operatorname{lk}_{\Delta} v_{k}$ is a homology $(d-3)$-sphere.

Proof. Let $\{i, j, k\}=[3]$ be distinct, $\Sigma=\mathrm{lk}_{\Delta} v_{i} \cap \mathrm{lk}_{\Delta} v_{j}$ and $\Gamma=\cap_{k=1}^{3} \mathrm{lk}_{\Delta} v_{k}$. We first prove that $\Sigma$ and $\Gamma$ have the same homology as a simplicial $(d-2)$-ball and simplicial $(d-3)$-sphere respectively. Since each $(d-2)$-face of $\Delta$ is contained in exactly 2 facets, it follows that $\operatorname{lk}_{\Delta} v_{i} \cup \mathrm{lk}_{\Delta} v_{j}=\Delta_{[d-1]}$. By the Mayer-Vietoris sequence, for any $n \geqslant 0$,

$$
\cdots \rightarrow H_{n+1}\left(\Delta_{[d-1]}\right) \rightarrow H_{n}(\Sigma) \rightarrow H_{n}\left(\mathrm{lk}_{\Delta} v_{i}\right) \oplus H_{n}\left(\mathrm{lk}_{\Delta} v_{j}\right) \rightarrow H_{n}\left(\Delta_{[d-1]}\right) \rightarrow \cdots .
$$

Note that $\Delta_{[d-1]}$ is a deformation retract of $\Delta$ minus three points, hence $\beta_{d-2}\left(\Delta_{[d-1]}\right)=2$ and $\beta_{k}\left(\Delta_{[d-1]}\right)=0$ for $0 \leqslant k \leqslant d-3$. We conclude from (1) that $\beta_{k}(\Sigma)=0$ for all $k \geqslant 0$. Since $\mathrm{lk}_{\Delta} v_{k} \cup \Sigma=\Delta_{[d-1]}$ and $\mathrm{lk}_{\Delta} v_{k} \cap \Sigma=\Gamma$, by the Mayer-Vietoris sequence we obtain

$$
\cdots \rightarrow H_{n+1}\left(\Delta_{[d-1]}\right) \rightarrow H_{n}(\Gamma) \rightarrow H_{n}\left(\mathrm{lk}_{\Delta} v_{k}\right) \oplus H_{n}(\Sigma) \rightarrow H_{n}\left(\Delta_{[d-1]}\right) \rightarrow \cdots
$$

Hence $\beta_{d-3}(\Gamma)=1$ and $\beta_{k}(\Gamma)=0$ for $0 \leqslant k \leqslant d-4$.

Next, for any $\tau \in \Gamma$, we have $\operatorname{lk}_{\Sigma} \tau=\operatorname{lk}_{\mathrm{lk}_{\Delta} \tau} v_{i} \cap \mathrm{lk}_{\mathrm{lk}_{\Delta} \tau} v_{j}$ and $\operatorname{lk}_{\Gamma} \tau=\cap_{i=1}^{3} \operatorname{lk}_{\mathrm{lk}_{\Delta} \tau} v_{i}$. Since $\mathrm{lk}_{\Delta} \tau$ is a balanced homology $(d-1-|\tau|)$-sphere, using the same argument as above, we may show that $l k_{\Sigma} \tau$ and $l k_{\Gamma} \tau$ have the same homology as a $(d-2-|\tau|)$-ball and $(d-3-|\tau|)$-sphere respectively. Therefore $\Gamma$ is a homology $(d-3)$-sphere. Finally, for any interior face $\sigma$ of $\Sigma, \mathrm{lk}_{\Sigma} \sigma=\mathrm{lk}_{\mathrm{lk}_{\Delta} v_{i}} \sigma=\mathrm{lk}_{\mathrm{lk}_{\Delta} v_{j}} \sigma$, and hence $\mathrm{lk}_{\Sigma} \sigma$ is a homology sphere. By definition we conclude that $\Sigma$ is a homology $(d-2)$-ball.

Remark 5. The complex $\Gamma$ in Lemma 4 is not balanced, since $\Gamma$ is $(d-1)$-colorable instead of being $(d-2)$-colorable.

Proposition 6. The only balanced 2-neighborly 3-manifold with 12 vertices triangulates the nonorientable $\mathbb{S}^{2}$-bundle over $\mathbb{S}^{1}$.

Proof. Let $\Delta$ be a balanced 2-neighborly 3-manifold with 12 vertices. Its $f$-vector is $f(\Delta)=(1,12,54,84,42)$. By Lemma 2 and Remark 3 , each color set of $\Delta$ has three 
vertices. We let $V_{4}=\left\{v_{1}, v_{2}, v_{3}\right\}$ be the set of vertices of color 4 . Since $\Delta$ is balanced 2-neighborly, each $\mathrm{lk}_{\Delta} v_{i}$ is a 2 -sphere with 9 vertices, its $f$-vector is $(1,9,21,14)$. Furthermore, the balancedness of $\Delta$ implies that every vertex $u \in \operatorname{lk}_{\Delta} v_{i}$ has $\operatorname{deg}_{\mathrm{lk}_{\Delta} v_{i}} u=4$ or 6 . If $x$ is the number of vertices of degree 6 in $\mathrm{lk}_{\Delta} v_{i}$, then

$$
4(9-x)+6 x=\sum_{u \in \mathrm{lk}_{\Delta} v_{i}} \operatorname{deg}\left(\operatorname{lk}_{\mathrm{lk}_{\Delta} v_{i}} u\right)=2 f_{1}\left(\mathrm{lk}_{\Delta} v_{i}\right)=42,
$$

and hence $x=3$. A balanced 2-sphere with 9 vertices, 3 of which have degree 6 , is unique up to isomorphism, as shown in Figure 1. Hence all vertex links in $\Delta$ are combinatorially isomorphic.

Since $f_{i}\left(\Delta_{[3]}\right)=f_{i}(\Delta)-\sum_{j=1}^{3} f_{i-1}\left(\mathrm{lk}_{\Delta} v_{j}\right)$ for all $i \leqslant 2$, we have $f\left(\Delta_{[3]}\right)=(1,9,27,21)$. Let $i, j, k \in[3]$ be distinct and $\Sigma:=\mathrm{lk}_{\Delta} v_{i} \cap \mathrm{lk}_{\Delta} v_{j}$. Any facet $F$ of $\Sigma$ are 2-dimensional, for otherwise if $F$ is an edge, then $\mathrm{lk}_{\Delta} F$ is either a 4-cycle or 6-cycle, where in both cases $v_{i}$ and $v_{j}$ share at least one common neighbor $w$, i.e., $F \cup\{w\} \in \Sigma$, a contradiction. Similarly, the facet cannot be 0-dimensional. Also the facets of $\Sigma$ do not belong to $\mathrm{lk}_{\Delta} v_{k}$. Hence $\Sigma$ is a pure 2-dimensional subcomplex of $\mathrm{lk}_{\Delta} v_{i}$ with 9 vertices and $f_{2}\left(\Delta_{[3]}\right)-f_{2}\left(\mathrm{lk}_{\Delta} v_{k}\right)=7$ triangles.

On the other hand, for any $u \in \Sigma$, the vertex link $\mathrm{lk}_{\Delta} u$ is isomorphic to Figure 1 and $v_{i}, v_{j} \in \mathrm{lk}_{\Delta} u$. The intersection of links of arbitrary two vertices of the same color has the following property

$(*): \quad \quad \quad \mathrm{k}_{\Sigma} u=\operatorname{lk}_{\mathrm{lk}_{\Delta} u} v_{i} \cap \mathrm{lk}_{\mathrm{lk}_{\Delta} u} v_{j}$ is either an edge, or a path of length 3.

Since $\Sigma$ is 2-dimensional, each connected component of $\Sigma$ has at least 3 vertices. If there are three components, then $\Sigma$ is the disjoint union of three triangles, contradicting that $f_{2}(\Sigma)=7$. Otherwise, if $\Sigma$ is connected, then by observation $(*)$ we have that $\Sigma$ is a triangulated 2-manifold (with boundary). Since $21=3 f_{2}(\Sigma)=\sum_{u \in \Sigma} f_{1}\left(\mathrm{lk}_{\Sigma} u\right)$ and by observation $(*) f_{1}\left(\mathrm{lk}_{\Sigma} u\right) \in\{1,3\}$, the links of three vertices in $\Sigma$ are single edges, while the rest are paths of length 3 . However, enumeration based on observation $(*)$ yields that there is no such complex $\Sigma$.

The last case is that $\Sigma$ has two connected components. From observation $(*)$ we see that each component cannot have 4 or 5 vertices. If one component is the triangle, then the other component (as a 6-triangle subcomplex of the 9-vertex balanced 2-sphere) must be the triangulated annulus as shown in Figure 1. By symmetry $\mathrm{lk}_{\Delta} v_{i} \cap \mathrm{lk}_{\Delta} v_{k}$ and $\mathrm{lk}_{\Delta} v_{j} \cap \mathrm{lk}_{\Delta} v_{k}$ are also isomorphic to $\Sigma$. In this way we determine $\Delta_{[3]}=\mathrm{lk}_{\Delta} v_{i} \cup$ $\mathrm{lk}_{\Delta} v_{j}$ : it is the union of three octahedral 2-spheres, each having a pair of antipodal facets $\left(F_{1}, F_{2}\right),\left(F_{2}, F_{3}\right)$ and $\left(F_{3}, F_{1}\right)$, respectively. This also determines $\Delta$, which is the balanced triangulation of the nonorientable $\mathbb{S}^{2}$-bundle over $\mathbb{S}^{1}$ known as ${ }^{3} 12_{2}^{83}$ in [10].

Remark 7 . The balanced 2-neighborly manifold ${ }^{3} 12_{2}^{83}$ is also known as $B M_{4}$ defined in [11]. In particular in [11, Proposition 6.9] it is shown that ${ }^{3} 12_{2}^{83}$ is the only balanced 12-vertex 3-manifold with $\beta_{1} \neq 0$. See [11] and [23] for extension in higher dimensional cases.

Next we characterize all balanced 3-spheres with each color sets of size 3 . 
Lemma 8. Up to an isomorphism, there are four triangulations of balanced 3-spheres with each color set of size 3 .

Proof. Let $\Delta$ be such a sphere and let $V_{4}=\left\{v_{1}, v_{2}, v_{3}\right\}$. Each vertex link of $\Delta$ is a balanced 2-sphere with at most 9 vertices, hence it is either the octahedral sphere, the suspension of a 6-cycle, or the connected sum of two octahedral spheres. We denote these three 2-spheres as $\Sigma_{1}, \Sigma_{2}$ and $\Sigma_{3}$ respectively. By Lemma $4, \Delta_{[3]}$ is the union of three triangulated 2-balls $B_{i}=\mathrm{lk}_{\Delta} v_{j} \cap \mathrm{lk}_{\Delta} v_{k}$, where $\{i, j, k\}=[3]$, glued along their common boundary complex $c$. Assume that $f_{0}\left(\mathrm{lk}_{\Delta} v_{i}\right) \leqslant f_{0}\left(\mathrm{lk}_{\Delta} v_{j}\right)$ when $i \leqslant j$. An easy counting leads to

$f_{0}\left(\Delta_{[3]}\right)=f_{0}(c)+\sum_{i=1}^{3} f_{0}\left(B_{i} \backslash c\right)=9, \quad f_{0}\left(\mathrm{lk}_{\Delta} v_{i}\right)=f_{0}(c)+f_{0}\left(B_{j} \backslash c\right)+f_{0}\left(B_{k} \backslash c\right) \in\{6,8,9\}$,

where $f_{0}\left(B_{i} \backslash c\right)$ counts the number of interior vertices of $B_{i}$. By the Dehn-Sommerville relations, the $f$-vector of any triangulated 3-manifold satisfies that $f_{1}=f_{3}+f_{0}$. Since every facet of $\Delta$ contains exactly one vertex from $V_{4}$, we have that $f_{3}(\Delta)=\sum_{i=1}^{3} f_{2}\left(\mathrm{lk}_{\Delta} v_{i}\right)$ and hence

$$
f_{1}(\Delta)=\sum_{i=1}^{3} f_{2}\left(\mathrm{lk}_{\Delta} v_{i}\right)+f_{0}(\Delta)=\sum_{i=1}^{3}\left(2 f_{0}\left(\mathrm{lk}_{\Delta} v_{i}\right)-4\right)+12=2 \sum_{i=1}^{3} f_{0}\left(\mathrm{lk}_{\Delta} v_{i}\right) \leqslant 54
$$

we enumerate the combinatorial type of each $f_{0}\left(\mathrm{lk}_{\Delta} v_{i}\right)$ as follows:

Case 1: $\mathrm{lk}_{\Delta} v_{1} \cong \Sigma_{1}$. It follows that $\mathrm{lk}_{\Delta} v_{3}$ is obtained from $\mathrm{lk}_{\Delta} v_{2}$ by a cross flip (see [5] for a reference). Since $f_{0}\left(\Delta_{[3]}\right)=9$, either $\mathrm{lk}_{\Delta} v_{2} \cong \Sigma_{1}, \mathrm{lk}_{\Delta} v_{3} \cong \Sigma_{3}$, and the cross flip replaces a 2-face of $\mathrm{lk}_{\Delta} v_{2}$ with its complement in the octahedral sphere. Or $\mathrm{lk}_{\Delta} v_{2} \cong \Sigma_{2}$, $\mathrm{lk}_{\Delta} v_{3} \cong \Sigma_{3}$, and the cross flip replaces the union of three 2 -faces of $\mathrm{lk}_{\Delta} v_{2}$ with its complement in the octahedral sphere. In the first case the 3 -sphere is the connected sum of two octahedral 3-spheres, which we denote as $S_{1}$. In the second case we obtain a 3sphere $S_{2}\left(\right.$ with $\mathrm{lk}_{S_{2}} v_{i} \cong \Sigma_{i}$ for $\left.i \in[3]\right)$. Their $f$-vectors are $f\left(S_{1}\right)=(1,12,42,60,30)$ and $f\left(S_{2}\right)=(1,12,46,68,34)$.

Case 2: $\operatorname{lk}_{\Delta} v_{1} \cong \Sigma_{2}$. In this case the number of missing colored edges in $\Delta_{[3]}$ is $\frac{9 \cdot 6}{2}-f_{1}\left(\Delta_{[3]}\right)=27-\sum_{i=1}^{3} f_{0}\left(\mathrm{lk}_{\Delta} v_{i}\right)$, which equals either 1,2 or 3 .

Subcase 1: $\mathrm{lk}_{\Delta} v_{2} \cong \mathrm{lk}_{\Delta} v_{3} \cong \Sigma_{2}$. By conditions (2), $c$ is a 6-cycle and $\Delta_{[3]} \backslash c$ consists of three disjoint vertices of degree either 4 or 6 . Note that in $\Sigma_{2}$ every pair vertices of degree 4 and degree 6 forms an edge. Since $\Delta_{[3]}$ has only three missing edges between vertices of different colors, the vertices in $\Delta_{[3]} \backslash c$ are of the same color and has degree 6 . Hence $\Delta_{[3]}$ is the join of $c$ and three disjoint vertices and $\Delta$ is the join of two 6-cycles. Denote this sphere as $S_{3}$; its $f$-vector is $(1,12,48,72,36)$.

Subcase 2: $\operatorname{lk}_{\Delta} v_{i} \cong \Sigma_{i}$ for $i=2,3$. Then $c$ is a 7 -cycle; furthermore, $B_{3}$ has no interior vertices and $B_{1}, B_{2}$ have a unique interior vertex $b_{1}, b_{2}$ respectively. Since $\Delta_{[3]}$ has two missing colored edges, and three vertices of degree 6 form an empty triangle in $\mathrm{lk}_{\Delta} v_{3} \cong \Sigma_{3}$, WLOG assume that $\operatorname{deg} b_{1}=4$ and $\operatorname{deg} b_{2}=6$. The only vertex $b_{3}$ not connected to $b_{2}$ in $B_{2}$ must be the vertex of degree 6 in $\mathrm{lk}_{\Delta} v_{1} \cong \Sigma_{2}$. Hence $B_{3}$ is the join 
of $b_{3}$ with the path of length 5 , which is $c \backslash b_{3}$. But then $b_{3}$ is also the degree- 6 vertex in $\mathrm{lk}_{\Delta} v_{2} \cong \Sigma_{2}$, and there is no way to triangulate $B_{1}$ such that it shares no common interior edge with $B_{2}$.

Subcase 3: $\mathrm{lk}_{\Delta} v_{2} \cong \mathrm{lk}_{\Delta} v_{3} \cong \Sigma_{3}$. Then $c$ is an 8-cycle and only $B_{1}$ has an interior vertex $a$. Also $\Delta_{[3]}$ has one missing colored edge, so $a$ is of degree 6 . Three vertices of degree 6 in $\Sigma_{3}$ are of different colors, hence in $l_{k_{\Delta}} v_{2}$ and $l_{k_{\Delta}} v_{3}$ the other two vertices of degree 6 must be two pairs of antipodal vertices in $\mathrm{lk}_{\Delta_{[3]}} a$ (the other pair of antipodal vertices in $\mathrm{lk}_{\Delta_{[3]}} a$ is the missing edge in $\left.\Delta_{[3]}\right)$. In this way we recover $1 \mathrm{k}_{\Delta} v_{2}=B_{1} \cup$ $B_{3}, \mathrm{lk}_{\Delta} v_{3}=B_{1} \cup B_{2}$, where $B_{1}, B_{2}, B_{3}$ are the 2-balls shown in the figure below (from left to right); in particular, labels of the vertices represent the color and the blue (red resp.) edges form the missing triangle in $\mathrm{lk}_{\Delta} v_{2}$ ( $\mathrm{lk}_{\Delta} v_{3}$ resp.). We call this 3 -sphere $S_{4}$. Its $f$-vector is $(1,12,52,80,40)$.

Case 3: $\mathrm{lk}_{\Delta} v_{1} \cong \Sigma_{3}$. This is not possible by Proposition 6 .
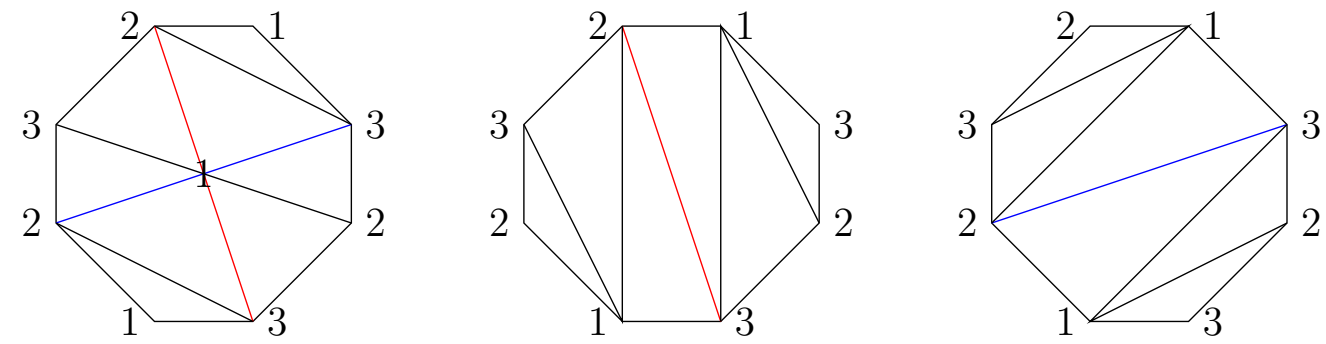

Theorem 9. There exists a unique balanced 2-neighborly homology 4-sphere ${ }^{4} 15_{2}^{5}$ with each color set of size 3 .

Proof. Let $\Delta$ be such a sphere and let its color set $V_{5}=\left\{v_{1}, v_{2}, v_{3}\right\}$. By Alexander Duality, $\tilde{H}_{i}\left(\Delta_{\{4,5\}}\right) \cong \tilde{H}_{3-i}\left(\Delta_{[3]}\right)$. In particular, since $\Delta_{\{4,5\}}$ is balanced 2-neighborly, $\beta_{2}\left(\Delta_{[3]}\right)=\beta_{1}\left(\Delta_{\{4,5\}}\right)=4$ and $\beta_{1}\left(\Delta_{[3]}\right)=0$. Hence

$$
f_{2}\left(\Delta_{[3]}\right)=\left(f_{1}-f_{0}+\chi\right)\left(\Delta_{[3]}\right)=\frac{9 \cdot 6}{2}-9+5=23 .
$$

By double counting, $\sum_{i=1}^{3} f_{1}\left(\mathrm{lk}_{\Delta} v_{i}\right)=\sum_{W=\{i, j, 5\} \subseteq[5]} f_{2}\left(\Delta_{W}\right)=\left(\begin{array}{l}4 \\ 2\end{array}\right) f_{2}\left(\Delta_{[3]}\right)=138$. By Proposition 6 and Lemma $8, f_{1}\left(\mathrm{lk}_{\Delta} v_{i}\right) \in\{42,46,48,52\}$, it follows that either $138=$ $42+48 \cdot 2$, that is, $\mathrm{lk}_{\Delta} v_{1} \cong S_{1}$ and $\mathrm{lk}_{\Delta} v_{2}, \mathrm{lk}_{\Delta} v_{3} \cong S_{3}$; or $138=46 \cdot 3$ and $\mathrm{lk}_{\Delta} v_{i} \cong S_{2}$ for all $i$.

Consider the first case above. $S_{1}$ is the connected sum of two octahedral 3-spheres. For any 2-subset $W \subset[4]$, the induced subcomplex $\left(S_{1}\right)_{W}$ is the union of two 4-cycles glued along an edge, so $f_{1}\left(\left(S_{1}\right)_{W}\right)=7$. Similarly, $S_{3}$ is the join of two 6-cycles, so we have $\left(S_{3}\right)_{W}$ is either a 6 -cycle or the bipartite graph $K_{3,3}$, i.e., $f_{1}\left(\left(S_{3}\right)_{W}\right)=6$ or 9 . Hence

$$
23=f_{2}\left(\Delta_{W \cup\{5\}}\right)=\sum_{i=1}^{3} f_{1}\left(\left(\mathrm{lk}_{\Delta} v_{i}\right)_{W}\right) \in\{19,22,25\},
$$


a contradiction.

Now we consider the second case, where all vertex links in $\Delta$ are isomorphic to $S_{2}$. Let $\Gamma=\mathrm{lk}_{\Delta} v_{1} \cap \mathrm{lk}_{\Delta} v_{2} \cap \mathrm{lk}_{\Delta} v_{3}$. The proof of Lemma 8 implies that for any vertex $p \notin V_{5}$, $\mathrm{lk}_{\Gamma} p=\mathrm{lk}_{\mathrm{lk}_{\Delta} p} v_{1} \cap \mathrm{lk}_{\mathrm{lk}_{\Delta} p} v_{2} \cap \mathrm{lk}_{\mathrm{lk}_{\Delta} p} v_{3}$ is a 5-cycle (as the boundary of the union of three 2 -faces, where we apply the cross-flip). Hence $\Gamma$ must be the boundary of the icosahedron. Since all $\mathrm{lk}_{\Delta} v_{i}$ are isomorphic, by Lemma $4 \Gamma$ divides the 3 -sphere $\mathrm{lk}_{\Delta} v_{1}$ into two 3 -balls, each having the same number of facets. If the facets of $\mathrm{lk}_{\Delta} v_{1}$ are labeled as in the link of vertex 1 in ${ }^{4} 15_{2}^{5}$ (this is a vertex-transitive triangulation of 4 -sphere whose vertex links are isomorphic to $S_{2}$, see [10]), then one such $\Gamma$ is the intersection of vertices $1,6,8$ in ${ }^{4} 15_{2}^{5}$; we rename it to $\Gamma_{1}$. In this case, $\mathrm{lk}_{\Delta} v_{1}=B \cup_{\Gamma_{1}} B^{\prime}$, where $B, B^{\prime}$ are isomorphic 3-balls. We check by sage [21] that all other subcomplexes in $l_{k_{\Delta}} v_{1}$ that are isomorphic to $\Gamma_{1}$ are of the form $\sigma\left(\Gamma_{1}\right)$, where $\sigma$ is an element in the permutation group of $\mathrm{lk}_{\Delta} v_{1}$ (of order 8). So it suffices to consider just $\Gamma_{1}$. To reconstruct $l_{\mathrm{k}_{\Delta}} v_{2}$ and hence $\Delta$, note that $\mathrm{lk}_{\Delta} u_{2}$ has the decomposition $\mathrm{lk}_{\Delta} u_{2}=B^{\prime} \cup_{\Gamma_{1}} B^{\prime \prime} \cong S_{2}$ for some 3 -ball $B^{\prime \prime}$, and furthermore $B^{\prime \prime} \cong B^{\prime}$. To decide $B^{\prime \prime}$ it is equivalent to finding a balanced simplicial isomorphism $f: B^{\prime} \rightarrow B^{\prime \prime}$ with $B^{\prime} \cap B^{\prime \prime}=B \cap B^{\prime \prime}=\Gamma_{1}$ and $f\left(\Gamma_{1}\right)=\Gamma_{1}$; in other words, $f$ is a permutation in Aut $\left(\Gamma_{1}\right)$. We check by sage $[21]$ that the links of vertex 6,8 in ${ }^{4} 15_{2}^{5}$ are the only candidates for $\mathrm{lk}_{\Delta} v_{2}$. Hence $\Delta={ }^{4} 15_{2}^{5}$. Indeed $\Delta$ is balanced: the color sets are $\{1,6,8\},\{2,4,9\}$, $\{3,7,11\},\{5,10,15\}$ and $\{12,13,14\}$.

Theorem 10. There exists a balanced 3-neighborly non-spherical 5-manifold with each color set of size 3 .

Proof. By Theorem 9, if such 5-manifold exists, then all vertex links are isomorphic to ${ }^{4} 15_{2}^{5}$, which we denote as $\Gamma$. Based on the list of facets of $\Gamma$ in [15], we take a colorpreserving permutation $\sigma=(1,6,8)(2,4,9)(11,3,7)(10,15,5)(13,14,12)$. We choose $\sigma$ in such a way that $\sigma \notin \operatorname{Aut}(\Gamma)$ and furthermore, $\Gamma \cap \sigma(\Gamma), \Gamma \cap \sigma^{2}(\Gamma)$ and $\sigma(\Gamma) \cap \sigma^{2}(\Gamma)$ are isomorphic homology manifolds with no interior faces of dimension $<2$ and with a common boundary $C$. By computer we check that $\Gamma \cup \sigma(\Gamma) \cup \sigma^{2}(\Gamma)$ is balanced 3-neighborly and $C$ is the vertex-transitive 3 -manifold ${ }^{3} 15_{1}^{15}$ that triangulates $\mathbb{S}^{3} / Q$ as in [15]. Finally let $\Delta=(\Gamma *\{16\}) \cup(\sigma(\Gamma) *\{17\}) \cup\left(\sigma^{2}(\Gamma) *\{18\}\right)$, where $\{16,17,18\}$ are the vertices of color 6 . By sage [21] one verifies that all vertex links of $\Delta$ is isomorphic to $\Gamma$, which is known as a combinatorial 4-sphere (we say a simplicial complex is a combinatorial sphere if it is PL homeomorphic to the boundary of the simplex). Hence $\Delta$ is a combinatorial manifold that is balanced 3-neighborly.

Remark 11. The following properties of the balanced 3-neighborly 5-manifold found in the proof of Theorem 10 are verified by sage:

1. It is vertex-transitive and has the following generators of the automorphism group (of order 1080):

$$
\begin{gathered}
(2,15)(4,5)(9,10)(12,17)(13,18)(14,16),(1,2)(3,15)(4,6)(5,7)(8,9)(10,11), \\
(1,3)(6,7)(8,11)(12,17)(13,18)(14,16),(1,13)(3,18)(6,14)(7,16)(8,12)(11,17), \\
(1,6,8)(2,3,17)(4,7,18)(5,15,10)(9,11,16) .
\end{gathered}
$$


2. The homology groups of $\Delta$ are given by $\left(\mathbb{Z}, 0, \mathbb{Z}_{2}, 0,0, \mathbb{Z}\right)$.

3. The $f$-vector of $\Delta$ is $(1,18,135,540,1035,918,306)$.

Furthermore by the Dehn-Sommerville relations, any balanced 3-neighborly 5-manifold having 3 vertex in each color set also has the $f$-vector $(1,18,135,540,1035,918,306)$. Let $\Sigma$ be such a complex, $\left\{v_{1}, v_{2}, v_{3}\right\}$ a color set, $M_{i}=\operatorname{lk}_{\Sigma} v_{j} \cap \mathrm{lk}_{\Sigma} v_{k}$ for $\{i, j, k\}=[3]$ and $N=M_{1} \cap M_{2} \cap M_{3}$. If $F$ is an interior face of $M_{i}$, then $F \cup\left\{v_{i}\right\} \notin \Sigma$. Since $\Sigma$ is balanced 3-neighborly, $\mathrm{lk}_{\Sigma} v_{1}=M_{2} \cup M_{3} \cong M_{1} \cup M_{3}=\mathrm{lk}_{\Sigma} v_{2}$ and they have 102 facets, it follows that each $M_{i}$ has no interior vertices or edges and with 51 facets. Also since all vertex links in $\Sigma$ are isomorphic to ${ }^{4} 15_{2}^{5}$, the same argument as in the proof of Theorem 9 implies that $N$ is a 3 -manifold whose vertex links are isomorphic to the boundary of the icosahedron; indeed ${ }^{3} 15_{1}^{15}$ is one such example. We haven't checked if there exist other balanced 3-neighborly 5-manifolds. (It is not known if there exist 15-vertex nonvertex-transitive 3 -manifolds whose vertex links are all isomorphic to the boundary of the icosahedron.)

\section{Balanced 2-neighborly 3 -sphere with 16 vertices}

In this section we provide a balanced 2-neighborly triangulation of the 3-sphere. The construction is motivated by Lemma 4 .
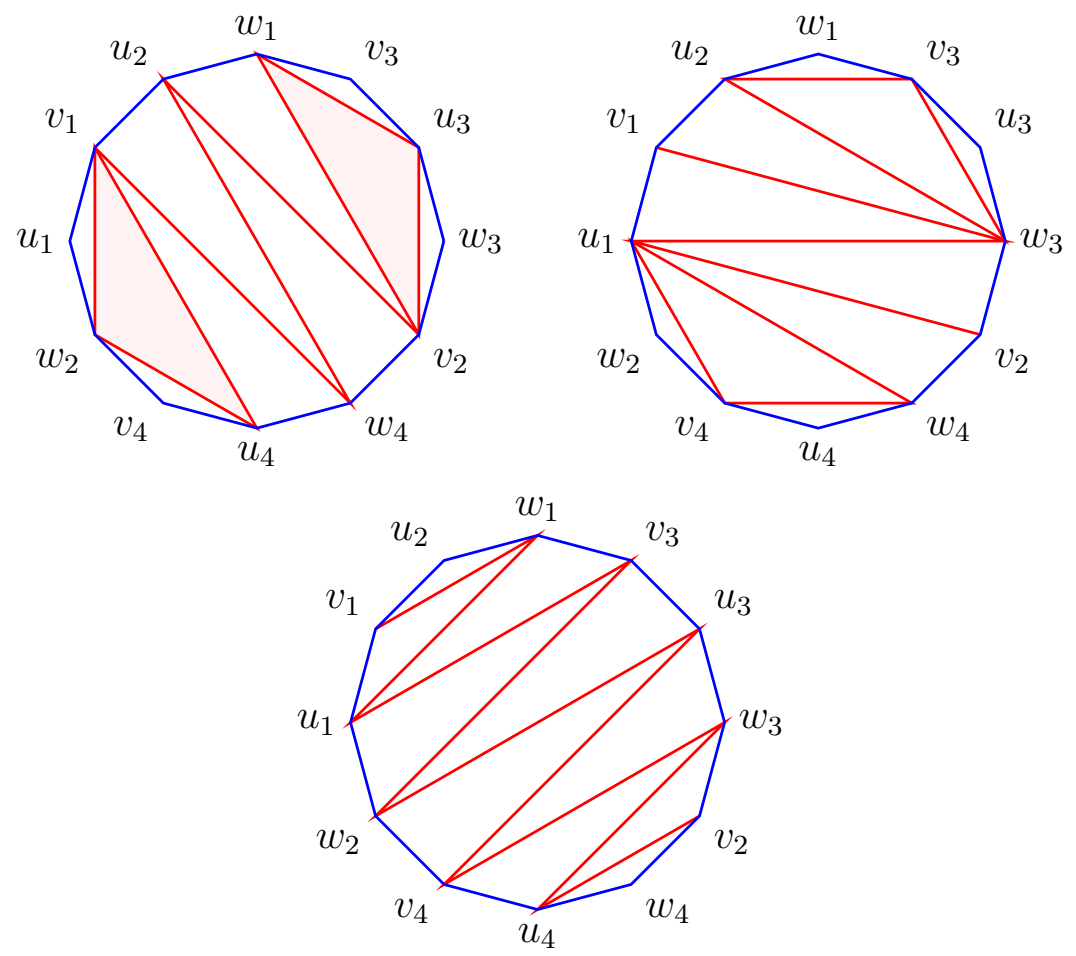

Figure 2: Discs $A, B$ and $C$ (from left to right) 

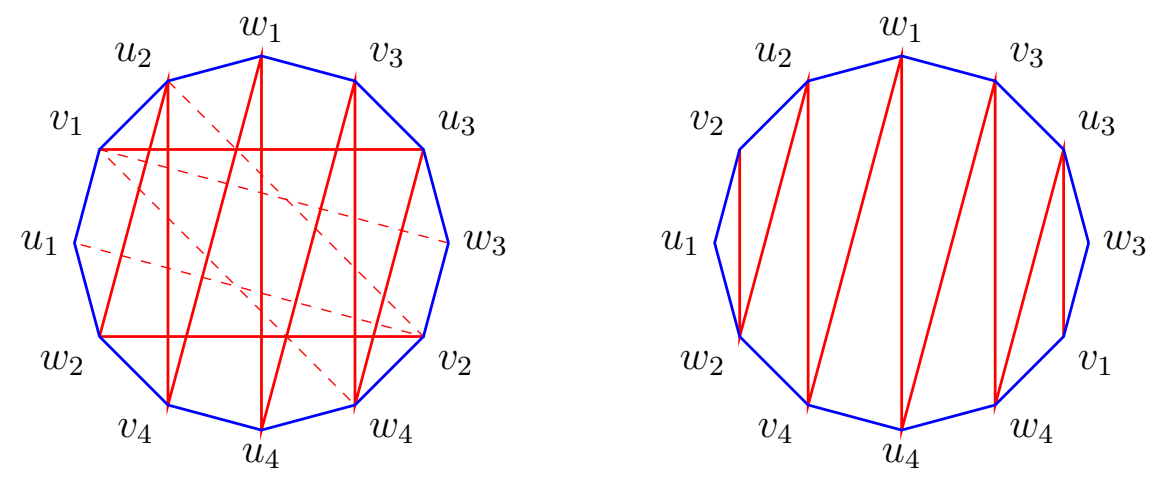

Figure 3: Left: disc $D^{\prime}$. Right: disc $D$ obtained after rearranging the boundary of $D^{\prime}$.
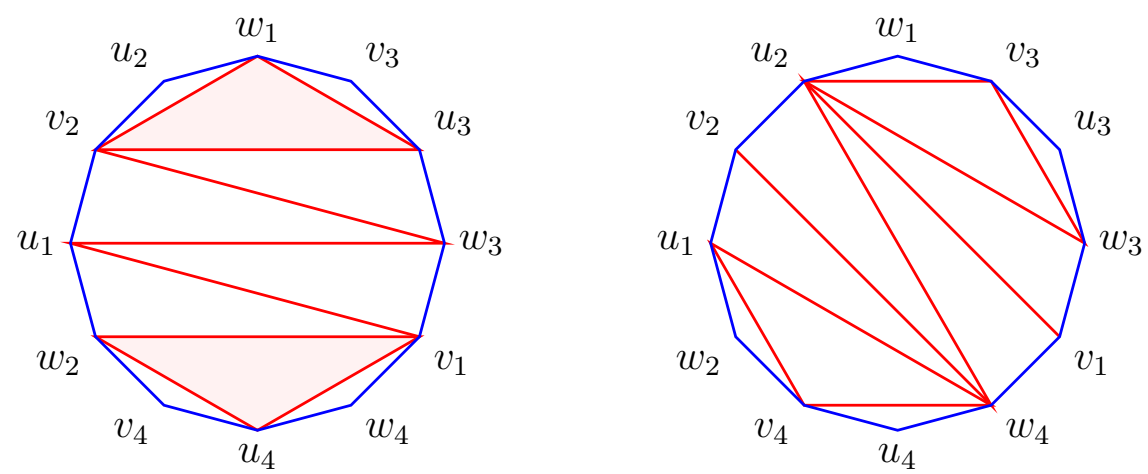

Figure 4: Left: $\operatorname{disc} A^{\prime}$. Right: $\operatorname{disc} B^{\prime}$. Notice that $\partial A^{\prime}=\partial B^{\prime}=\partial D$.

Construction 12. Assume $V_{1}=\left\{u_{1}, u_{2}, u_{3}, u_{4}\right\}, V_{2}=\left\{v_{1}, v_{2}, v_{3}, v_{4}\right\}, V_{3}=\left\{w_{1}, w_{2}, w_{3}, w_{4}\right\}$ and $V_{4}=\left\{z_{1}, z_{2}, z_{3}, z_{4}\right\}$ are the four color sets of a balanced 3 -sphere $\Gamma$. We let $\operatorname{lk}_{\Gamma} z_{1}=$ $A \cup_{\partial A \sim \partial C} C$ and $\operatorname{lk}_{\Gamma} z_{3}=B \cup_{\partial B \sim \partial C} C$, where $A, B$ and $C$ are triangulated 2-balls sharing the same boundary as shown in Figure 2. All possible edges that do not appear in $A, B$ and $C$ are shown in Figure 3 as solid red edges in disc $D^{\prime}$. Notice that the dashed edges in $D^{\prime}$ are edges in discs $A$ and $B$, so we may rearrange the boundary of $D$ by switching the positions of vertices $v_{1}$ and $v_{2}$, and then replacing the edges containing $v_{1}$ or $v_{2}$ in $\partial D^{\prime}$ by the dashed edges. In this way, we obtain a triangulation of a 12-gon $D$ as shown in Figure 3. Furthermore, $\partial D \subseteq A \cup B$, and $\partial D$ divides the sphere $=A \cup_{\partial A \sim \partial B} B$ into two discs $A^{\prime}$ and $B^{\prime}$ as shown in Figure 4.

We let $\mathrm{lk}_{\Gamma} z_{2}=A^{\prime} \cup_{\partial A^{\prime} \sim \partial D} D$ and $\mathrm{lk}_{\Gamma} z_{4}=B^{\prime} \cup_{\partial B^{\prime} \sim \partial D} D$. Since both $\mathrm{st}_{\Gamma} z_{1} \cap \mathrm{st}_{\Gamma} z_{3}=C$ and $\operatorname{st}_{\Gamma} z_{2} \cap\left(\operatorname{st}_{\Gamma} z_{1} \cup \mathrm{st}_{\Gamma} z_{3}\right)=A^{\prime}$ are simplicial 2-balls, it follows that $\Sigma=\cup_{i=1}^{3} \mathrm{st}_{\Gamma} z_{i}$ is a simplicial 3-ball. Furthermore, the boundary of $\Sigma$ is exactly $\operatorname{lk}_{\Gamma} z_{4}$. Hence $\Gamma=\Sigma \cup \mathrm{st}_{\Gamma} z_{4}$ is indeed a balanced 2-neighborly 3-sphere.

Remark 13. Here we provide some properties of $\Gamma$ in Construction 12.

1. $(A \cup B, C, D)$ is an ear decomposition of $\Gamma_{[3]}$. 
2. The automorphism group of $\Gamma$ has two generators

$$
\begin{gathered}
\left(u_{1} u_{3} u_{2} u_{4}\right)\left(v_{1} z_{2} v_{2} z_{1}\right)\left(v_{3} z_{4} v_{4} z_{3}\right)\left(w_{1} w_{4} w_{2} w_{3}\right), \\
\left(z_{1} v_{1}\right)\left(z_{2} v_{2}\right)\left(z_{3} v_{3}\right)\left(z_{4} v_{4}\right)\left(u_{1} w_{1}\right)\left(u_{2} w_{2}\right)\left(u_{3} w_{3}\right)\left(u_{4} w_{4}\right) .
\end{gathered}
$$

(The second generator is given by switching vertices of color 1 and 3, and color 2 and 4 , but with the same subscript.) Hence $\operatorname{Aut}(\Gamma)$ has 8 elements.

3. The complex $\Gamma$ given in Construction 12 is shellable. For $\mathrm{lk}_{\Gamma} z_{1}=A \cup_{\partial A \sim \partial C} C$, there exist two shellings $c_{1}, \ldots, c_{10}, a_{1}, \ldots, a_{10}$ and $a_{1}^{\prime}, \ldots, a_{10}^{\prime}, c_{1}^{\prime}, \ldots, c_{10}^{\prime}$ such that for any $1 \leqslant i \leqslant 10, c_{i}, c_{i}^{\prime}$ are facets from $C$ and $a_{i}, a_{i}^{\prime}$ are facets from $A$. Similarly, there exist two shellings $c_{1}, \ldots, c_{10}, b_{1}, \ldots, b_{10}$ and $b_{1}^{\prime}, \ldots, b_{10}^{\prime}, c_{1}^{\prime}, \ldots, c_{10}^{\prime}$ for $\operatorname{lk}_{\Gamma} z_{3}=B \cup_{\partial B \sim \partial C} C$, where $b_{i}, b_{i}^{\prime}$ are facets from $B$. Then

$$
a_{1}^{\prime} * z_{1}, \ldots, a_{10}^{\prime} * z_{1}, c_{1}^{\prime} * z_{1}, \ldots, c_{10}^{\prime} * z_{1}, c_{1} * z_{3}, \ldots, c_{10} * z_{3}, b_{1} * z_{3}, \ldots, b_{10} * z_{3}
$$

gives a shelling of $\operatorname{st}_{\Gamma} z_{1} \cup \mathrm{st}_{\Gamma} z_{3}$. We may extend this shelling into a complete shelling of $\Gamma$ by constructing two similar shellings of $\mathrm{lk}_{\Gamma} z_{2}$ and $\mathrm{lk}_{\Gamma} z_{4}$. However, we tried some computer tests and failed to prove either polytopality or non-polytopality.

Remark 14. It is easy to see that if $\Delta_{1}$ is a balanced 2-neighborly $\left(d_{1}-1\right)$-sphere and $\Delta_{2}$ is a balanced 2-neighborly $\left(d_{2}-1\right)$-sphere, then $\Delta_{1} * \Delta_{2}$ is a balanced 2-neighborly $\left(d_{1}+d_{2}-1\right)$-sphere. Hence by taking joins, we find balanced 2-neighborly $(4 k-1)$-spheres with $16 k$ vertices for any $k \geqslant 1$.

Question 15. Let $d \geqslant 4$ and $m \geqslant 5$ be arbitrary integers. Is there a balanced 2-neighborly simplicial $(d-1)$-sphere all of whose color sets have the same size $m$ ? Is there a polytopal sphere with these properties?

\section{Balanced 2-neighborly $L(3,1)$ with 16 vertices}

In this section we present our first construction of a balanced 2-neighborly lens space $L(3,1)$ with 16 vertices. We denote it by $\Delta$. Each color set of $\Delta$ has four vertices.

Construction 16. Denote the color sets of $\Delta$ by $V_{1}=\left\{u_{1}, u_{2}, u_{3}, u_{4}\right\}, V_{2}=\left\{v_{1}, v_{2}, v_{3}, v_{4}\right\}$, $V_{3}=\left\{w_{1}, w_{2}, w_{3}, w_{4}\right\}$ and $V_{4}=\left\{z_{1}, z_{2}, z_{3}, z_{4}\right\}$.

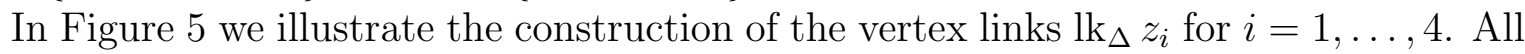
these links are realized as cylinders. Two links $\mathrm{lk}_{\Delta} z_{1}$ and $\mathrm{lk}_{\Delta} z_{2}$ share the same top and bottom, which are triangulated hexagons spanned by vertices $\left\{u_{i}, v_{i}, w_{i}: i=1,3\right\}$ and $\left\{u_{i}, v_{i}, w_{i}: i=2,4\right\}$, respectively. To construct $\mathrm{lk}_{\Delta} z_{3}$ from $\mathrm{lk}_{\Delta} z_{1}$, we switch the positions of vertices $u_{3}, v_{3}, w_{3}$ with vertices $u_{4}, v_{4}, w_{4}$ respectively and form a new cylinder. The new top and bottom hexagons contain the 2-faces $\left\{u_{1}, v_{1}, w_{1}\right\}$ and $\left\{u_{2}, v_{2}, w_{2}\right\}$. Similarly, we construct the link $\mathrm{lk}_{\Delta} z_{4}$ from $\mathrm{lk}_{\Delta} z_{2}$ by switching the positions of vertices $u_{3}, v_{3}, w_{3}$ with vertices $u_{4}, v_{4}, w_{4}$ and letting $\left\{u_{1}, v_{1}, w_{1}\right\}$ and $\left\{u_{2}, v_{2}, w_{2}\right\}$ be the 2-faces that appear in the triangulation of the top and bottom hexagons. It follows that $\mathrm{lk}_{\Delta} z_{3}$ and $\mathrm{lk}_{\Delta} z_{4}$ also share the same top and bottom. 


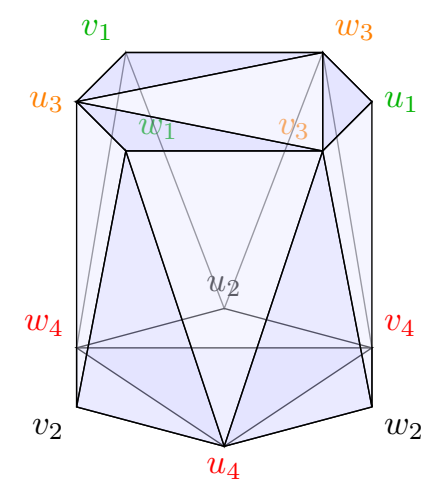

(a) $\mathrm{lk}_{\Delta} z_{1}$

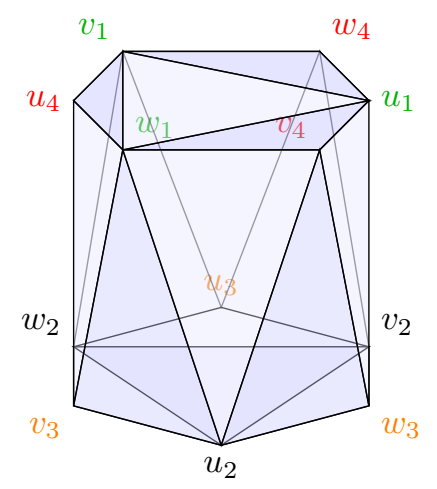

(c) $\mathrm{lk}_{\Delta} z_{3}$

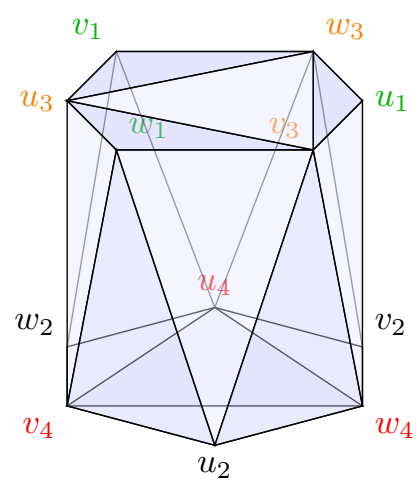

(b) $\mathrm{lk}_{\Delta} z_{2}$

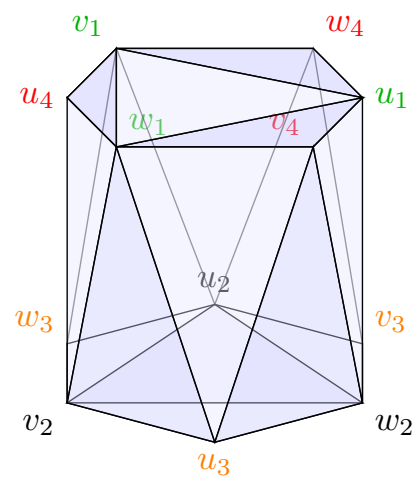

(d) $l \mathrm{k}_{\Delta} z_{4}$

Figure 5: Four vertex links of $\Delta$

Now since $\Delta$ is balanced 2-neighborly, by our construction, it only remains to show that $\Delta$ triangulates the lens space $L(3,1)$. The geometric realizations of st $z_{1}$ and st $z_{2}$ are filled cylinders that share top and bottom. So their union $A:=\mathrm{st}_{\Delta} z_{1} \cup \mathrm{st}_{\Delta} z_{2}$ is a filled torus (that is, a genus-1 handlebody); so is the union $B:=\operatorname{st}_{\Delta} z_{3} \cup \mathrm{st}_{\Delta} z_{4}$. Note that these two handlebodies have identical boundary complexes, thus they provide a Heegaard splitting of a lens space.

To identify which lens space $\Delta$ triangulates, we need to determine the homeomorphism $\phi: \partial A \rightarrow \partial B$. Consider two generators $\gamma, \delta$ of $\pi_{1}(A \cap B)=\pi_{1}(\partial A)$, where $\gamma$ is the 6cycle $\left(u_{3}, v_{1}, w_{3}, u_{1}, v_{3}, w_{1}\right)$ and $\delta$ is the 4 -cycle $\left(u_{1}, w_{2}, u_{4}, w_{3}\right)$. In particular, $\delta$ is also a generator of $\pi_{1}(A)$. From the construction we see that $\phi(\gamma)$ is a loop running around the equator of $\partial B$ thrice and the meridian of $\partial B$ once. Also $\phi(\delta)$ runs around the equator of $\partial B$ twice and the meridian of $\partial B$ once. Hence it is indeed the lens space $L(3,1)$.

Remark 17. Our construction $\Delta$ has the following properties:

1. All vertex links are combinatorially equivalent.

2. From Figure 5 we see $\mathrm{lk}_{\Delta} z_{i} \cap \mathrm{lk}_{\Delta} z_{j}$ has two connected components when $\{i, j\}=$ $\{1,2\}$ or $\{3,4\}$ (they are the top and bottom hexagons as shown in Figure 2); and it 
has three connected components when $i \in\{1,2\}$ and $j \in\{3,4\}$ (each component is the union of two facets along the side of the cylinders). In general, the intersection of two vertex links, where the vertices are of the same color, always has at least two connected components.

3. There are three group actions on the vertices of $\Delta$ :

(a) Fix the subscript and rotate the corresponding vertices of color 1, 2 and 3 respectively. The generator is given by $\left(u_{1} v_{1} w_{1}\right)\left(u_{2} v_{2} w_{2}\right)\left(u_{3} v_{3} w_{3}\right)$.

(b) Rotate vertices of the same color. The generator is

$$
\left(u_{1} u_{3} u_{2} u_{4}\right)\left(v_{1} v_{3} v_{2} v_{4}\right)\left(w_{1} w_{3} w_{2} w_{4}\right)\left(z_{1} z_{3} z_{2} z_{4}\right)
$$

(c) Exchange $\mathrm{lk}_{\Delta} z_{1}$ and $\mathrm{lk}_{\Delta} z_{2}, \mathrm{lk}_{\Delta} z_{3}$ and $\mathrm{lk}_{\Delta} z_{4}$, by exchanging $v_{i}$ and $w_{i}$ (or $u_{i}$ and $w_{i}, u_{i}$ and $v_{i}$ ) for all $i \in[4]$. The generators are

$$
\begin{aligned}
& \left(z_{1} z_{2}\right)\left(z_{3} z_{4}\right)\left(v_{1} w_{1}\right)\left(v_{2} w_{2}\right)\left(v_{3} w_{3}\right)\left(v_{4} w_{4}\right), \\
& \left(z_{1} z_{2}\right)\left(z_{3} z_{4}\right)\left(u_{1} w_{1}\right)\left(u_{2} w_{2}\right)\left(u_{3} w_{3}\right)\left(u_{4} w_{4}\right) \text { and } \\
& \left(z_{1} z_{2}\right)\left(z_{3} z_{4}\right)\left(u_{1} v_{1}\right)\left(u_{2} v_{2}\right)\left(u_{3} v_{3}\right)\left(u_{4} v_{4}\right) .
\end{aligned}
$$

The automorphism group of $\Delta$ is of size 96 .

Proposition 18. The complex $\Delta$ is a balanced vertex minimal triangulation of $L(3,1)$.

Proof. By Proposition 6.1 in [11], each color set of $\Delta$ is of size at least 3. If there are exactly three vertices $v_{1}, v_{2}, v_{3}$ of color 1 in $\Delta$, apply the Mayer-Vietoris sequence on the triple $\left(\mathrm{st}_{\Delta} v_{1} \cup \mathrm{st}_{\Delta} v_{2}, \mathrm{st}_{\Delta} v_{3}, \Delta\right)$ and we obtain that

$$
0=H_{1}\left(\mathrm{lk}_{\Delta} v_{3}\right) \rightarrow H_{1}\left(\mathrm{st}_{\Delta} v_{1} \cup \mathrm{st}_{\Delta} v_{2}\right) \oplus H_{1}\left(\mathrm{st}_{\Delta} v_{3}\right) \rightarrow H_{1}(\Delta) \rightarrow H_{0}\left(\mathrm{lk}_{\Delta} v_{3}\right)=0 .
$$

Hence $H_{1}\left(\right.$ st $_{\Delta} v_{1} \cup$ st $\left._{\Delta} v_{2}\right) \cong H_{1}(\Delta)=\mathbb{Z} / 3 \mathbb{Z}$. However, this is impossible since $H_{1}\left(\operatorname{st}_{\Delta} v_{1} \cup\right.$ st $\left._{\Delta} v_{2}\right) \cong H_{0}\left(\right.$ st $\left._{\Delta} v_{1} \cap \mathrm{st}_{\Delta} v_{2}\right)$, which cannot be $\mathbb{Z} / 3 \mathbb{Z}$.

The same argument as above also shows that the balanced triangulation of any lens space $L(p, q)$ with $p>1$ must have at least 16 vertices.

\section{Balanced spheres and ear decomposition}

In this section our goal is to construct a balanced 3-sphere whose rank-selected subcomplexes do not have ear decompositions. The motivation is from the balanced 2neighborly construction of $L(3,1)$ in Section 5 . Indeed, we want to construct a balanced 3-dimensional complex $\Delta$ so that 1) each vertex link is a 2 -sphere; 2) for a fixed color set $V_{4}=\left\{v_{1}, \cdots, v_{k}\right\}$, the intersection of any two vertex links $\mathrm{lk}_{\Delta} v_{i} \cap \mathrm{lk}_{\Delta} v_{j}$ always has at least two connected components (as the property listed in Remark 17); and 3) $\cup_{i=1}^{4} \mathrm{st}_{\Delta} v_{i}$ is 3 -ball, which together with the condition 1) guarantees that $\Delta$ is a 3 -sphere. 
In the following we take $k=5$ and give such a construction. Figure 6 illustrates the links $\mathrm{lk}_{\Delta} v_{1}, \cdots, \mathrm{lk}_{\Delta} v_{4}$. Every label represents the color of the vertex. Also each connected component of $\mathrm{lk}_{\Delta} v_{1} \cap \mathrm{lk}_{\Delta} v_{2}$ is colored in green, $\mathrm{lk}_{\Delta} v_{i} \cap \mathrm{lk}_{\Delta} v_{3}$ is colored in blue for $i=1,2$, and $\mathrm{lk}_{\Delta} v_{j} \cap \mathrm{lk}_{\Delta} v_{4}$ is colored in pink for $j=1,2,3$. Immediately we check that all these intersections of vertex links have 2 or 3 connected components.

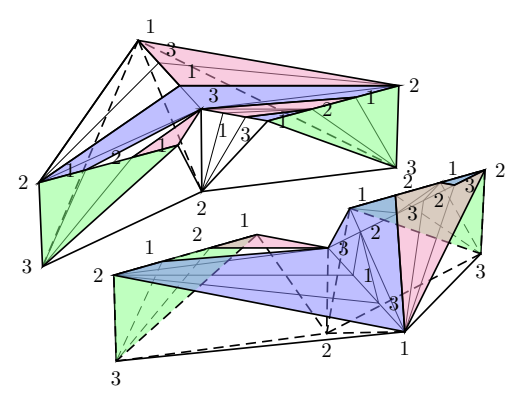

(a) $\mathrm{lk}_{\Delta} v_{1}$ and $\mathrm{lk}_{\Delta} v_{2}$

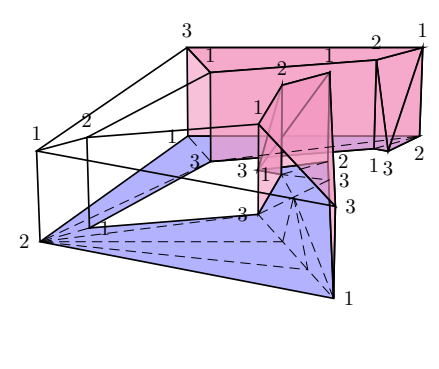

(b) $\mathrm{lk}_{\Delta} v_{3}$

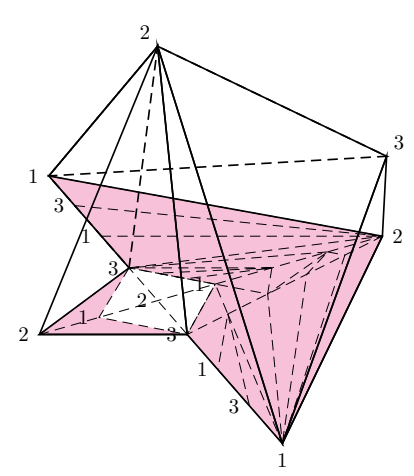

(c) $\mathrm{lk}_{\Delta} v_{4}$

Figure 6: Four vertex links as triangulated 2-spheres. For simplicity's sake, we omit some diagonal edges in the quadrilaterals in (b), and some labels of vertices in (c).

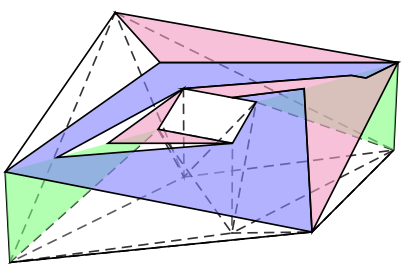

(a) $\mathrm{lk}_{\Delta} v_{1} \cup \mathrm{lk}_{\Delta} v_{2}$

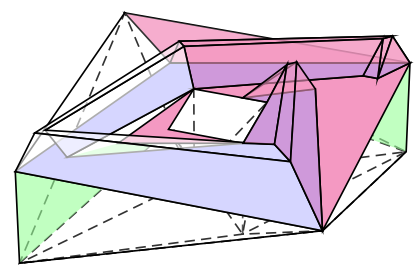

(b) $\cup_{i=1}^{3} \mathrm{lk}_{\Delta} v_{i}$

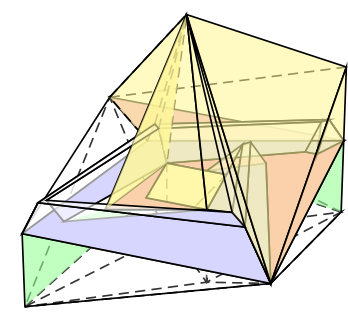

(c) $\cup_{i=1}^{4} \mathrm{lk}_{\Delta} v_{4}$

Figure 7: how the links are glued together.

Figure 7 shows how $\Delta \backslash V_{4}$ is formed from these links. First we glue $\mathrm{lk}_{\Delta} v_{1}$ and $\mathrm{lk}_{\Delta} v_{2}$ along two green triangles. The resulting complex $\mathrm{lk}_{\Delta} v_{1} \cup \mathrm{lk}_{\Delta} v_{2}$ is shown in Figure 7a. Then we place $\mathrm{lk}_{\Delta} v_{3}$ on top of $\mathrm{lk}_{\Delta} v_{1} \cup \mathrm{lk}_{\Delta} v_{2}$. As we see from Figure $7 \mathrm{~b}$, the boundary complex of $\cup_{i=1}^{3} \mathrm{st}_{\Delta} v_{i}$ is a triangulated torus. Finally, we place $\mathrm{lk}_{\Delta} v_{4}$ on top of $\cup_{i=1}^{3} \mathrm{lk}_{\Delta} v_{i}$ so that $\mathrm{st}_{\Delta} v_{4}$ "covers the 1-dimensional hole" in $\cup_{i=1}^{3} \mathrm{st}_{\Delta} v_{i}$, see Figure $7 \mathrm{c}$. We denote the subspace of $\mathbb{R}^{3}$ enclosed by $\mathrm{lk}_{\Delta} v_{i}$ as $S_{i}$ for $1 \leqslant i \leqslant 4$, and let $S_{5}:=\cup_{i \leqslant 4} S_{i}$. From our construction it follows that the boundary complex of $S_{5}$ is a 2 -sphere; we let it be $\operatorname{lk}_{\Delta} v_{5}$. 
Indeed $\Delta$ is a 3 -sphere since $\Delta$ is the union of two 3-balls $S_{5}$ and $\mathrm{st}_{\Delta} v_{5}$ glued along their common boundary $\mathrm{lk}_{\Delta} v_{5}$.

Since each $\mathrm{lk}_{\Delta} v_{i} \cap \mathrm{lk}_{\Delta} v_{j}$ has at least two connected components for $1 \leqslant i \neq j \leqslant 4$, the Mayer-Vietoris sequence implies that $S_{i} \cup S_{j}$ is not contractible for all $1 \leqslant i \neq j \leqslant 4$. A similar inspection of $\mathrm{lk}_{\Delta} v_{i} \cup \mathrm{lk}_{\Delta} v_{j} \cup \mathrm{lk}_{\Delta} v_{k}$ also implies that the boundary complexes of $S_{i} \cup S_{j} \cup S_{k}$ 's cannot be triangulated 2-spheres for distinct $1 \leqslant i, j, k \leqslant 4$.

Proposition 19. Not all rank-selected subcomplexes of balanced simplicial spheres have ear decompositions.

Proof. Consider the complex $\Delta$ constructed above. We denote the union of interior faces of a complex $\tau$ by int $\tau$. Suppose $\Delta \backslash V_{4}$ has an ear decomposition $\left(\Gamma_{1}, \Gamma_{2}, \cdots, \Gamma_{k}\right)$. Since $\left|V_{4}\right|=5$ and $\beta_{2}\left(\Delta \backslash V_{4}\right)=4, k$ must be 4 . Notice first that $\cup_{i \leqslant 4} l_{k_{\Delta}} v_{i}$ divides $\mathbb{R}^{3}$ into five subspaces, namely, $S_{1}, \cdots, S_{4}$ and the complement of $S_{5}$, each having $l_{k_{\Delta}} v_{i}$ as the boundary complex for $1 \leqslant i \leqslant 5$ respectively. The complex $\Gamma_{1}$ is the union of 2-balls $B_{1}, B_{2}$ with $\partial B_{1}=\partial B_{2}=\Gamma_{1} \cap \Gamma_{2}$. By the Jordan theorem, $B_{1} \cup \Gamma_{2}$ is a triangulated 2sphere that separates $\mathbb{R}^{3}$ into two connected components. Hence the bounded component must be either $S_{i} \cup S_{j}$ or $S_{i} \cup S_{j} \cup S_{k}$ for some $1 \leqslant i, j, k \leqslant 4$. (We may assume that it is not $S_{i}$, since otherwise we may consider the 2-sphere $\cup_{i \leqslant 3} \Gamma_{i}-\cup_{1 \leqslant i \neq j \leqslant 3} \operatorname{int}\left(\Gamma_{i} \cap \Gamma_{j}\right)$ instead of $\Gamma_{1} \cup \Gamma_{2}-\operatorname{int}\left(\Gamma_{1} \cap \Gamma_{2}\right)$, where the subset enclosed by this sphere in $\mathbb{R}^{3}$ cannot be $S_{i}$ anymore.) This contradicts the fact that the boundaries of $S_{i} \cup S_{j}$ or $S_{i} \cup S_{j} \cup S_{k}$ are not 2-spheres.

Remark 20. One can think of all the figures illustrated above as projections of a subcomplex of $\Delta-\mathrm{st}_{\Delta} v_{5}$ onto $\mathbb{R}^{3}$. However, we do not know whether the complex provided in this section can be realized as the boundary of a 4-polytope.

\section{Acknowledgements}

The author was partially supported by a graduate fellowship from NSF grant DMS1361423. I thank Moritz Firsching for pointing out the automorphism groups of the constructions in Section 3 and 4 and running some computational tests to decide whether the constructions are polytopal. Many thanks to Lorenzo Venturello and the anonymous referees for pointing out mistakes in an earlier version and contributing to a few remarks, improvement of the proofs in this paper.

\section{References}

[1] G. Burton. The non-neighbourliness of centrally symmetric convex polytopes having many vertices. J. Combin. Theory Ser. A, 58:321-322, 1991.

[2] M. K. Chari. Two decompositions in topological combinatorics with applications to matroid complexes. Trans. Amer. Math. Soc., 349:3925-3943, 1997.

[3] D. L. Donoho and J. Tanner. Counting the faces of randomly-projected hypercubes and orthants, with applications. Discrete Comput. Geom., 43:522-541, 2010. 
[4] B. Grünbaum. . Convex polytopes, Interscience, London, 1967.

[5] I. Izmestiev, S. Klee and I. Novik. Simplicial moves on balanced complexes. Advances in Mathematics, 320:82-114, 2017.

[6] M. Juhnke-Kubitzke, S. Murai, I. Novik and C. Sawaske. A generalized lower bound theorem for balanced manifolds. Mathematische Zeitschrift, 289:921-942, 2018.

[7] W. Jockusch. An infinite family of nearly neighborly centrally symmetric 3 -spheres. J. Combin. Theory. Ser. A, 72:318-321, 1995.

[8] M. Joswig and T. Rörig. Neighborly cubical polytopes and spheres. Israel J. Math., 159:221-242, 2007.

[9] M. Joswig and G. M. Ziegler. Neighborly cubical polytopes. Discrete Comput. Geom., 24:325-344, 2000.

[10] E. G. Köhler and F. Lutz. Triangulated Manifolds with Few Vertices: VertexTransitive Triangulations I. arXiv:math/0506520v1, 2005.

[11] S. Klee and I. Novik. Lower Bound Theorems and a Generalized Lower Bound Conjecture for balanced simplicial complexes. Mathematika, 62:441-477, 2016.

[12] P. McMullen. The maximum number of faces of a convex polytope. Mathematika, 17:179-184, 1970.

[13] M. Juhnke-Kubitzke and S. Murai. Balanced generalized lower bound inequality for simplicial polytopes. Selecta Math., 24:1677-1689, 2018.

[14] N. Linial and I. Novik. How neighborly can a centrally symmetric polytope be? Discrete Comput. Geom., 36:273-281, 2006.

[15] F. Lutz. https://page.math.tu-berlin.de/ lutz/stellar/4_manifolds.

[16] A. Padrol. Many neighborly polytopes and oriented matroids. Discrete Comput. Geom., 50:865-902, 2013.

[17] R. Sanyal and G. M. Ziegler. Construction and analysis of projected deformed products. Discrete Comput. Geom., 43:412-435, 2010.

[18] I. Shermer. Neighborly polytopes. Israel J. Math., 43:291-314, 1982.

[19] R. Stanley. The upper bound conjecture and Cohen-Macaulay rings. Studies in Applied Math., 54:135-142, 1975.

[20] R. Stanley. Balanced Cohen-Macaulay complexes. Trans. Amer. Math. Soc., 249:139157, 1979.

[21] William Stein et al. Sage Mathematics Software (Version 8.9), The Sage Development Team, 2019, http://www. sagemath.org.

[22] E. Swartz. g-elements, finite buildings and higher Cohen-Macaulay connectivity. J. Combin. Theory. Ser. A, 113:1305-1320, 2006.

[23] H. Zheng. Minimal balanced triangulations of sphere bundles over the circle. SIAM J. Disc. Math., 222:1259-1268, 2017. 